\section{Thrombotic thrombocytopenic purpura in a patient with interferon treated hepatitis C successfully treated with Rituximab}

\author{
Nishant Poddar, Jen C. Wang \\ Division of Hematology/Oncology, \\ Brookdale University Hospital Medical \\ Center, Brooklyn, NY, USA
}

\begin{abstract}
Thrombotic thrombocytopenic purpura (TTP) is a life threatening condition associated with formation of platelet thrombi. Deficiency of ADAM TS 13 with presence of inhibitory antiADAM TS 13 Immunoglobulin $\mathbf{G}$ antibody is seen in patients with acquired TTP. TTP in patients on interferon therapy for chronic hepatitis $\mathrm{C}$ has rarely been reported. Furthermore, successful treatment of an initial episode of acute refractory acquired TTP, in a patient of chronic hepatitis $\mathrm{C}$ during interferon therapy with Rituximab, has not been previously reported. Here we describe a case of acute refractory acquired TTP associated with pegylated interferon therapy for her chronic hepatitis $\mathrm{C}$ infection. Initially refractory to plasmapheresis and steroids, she was successfully treated with Rituximab and plasmaphersis without any evidence of reactivation of hepatitis.
\end{abstract}

\section{Introduction}

Thrombotic thrombocytopenic purpura (TTP) is a life threatening disease characterized by microangiopathic hemolytic anemia, thrombocytopenia, neurological disturbances, and renal failure. These symptoms are related to the presence of von Willebrand factor (VWF) - rich platelet thrombi in the arterioles and capillaries. $^{1}$

Most adult patients with acquired TTP have IgG autoantibodies that inhibit the von Willebrand factor-cleaving protease ADAMTS13 in plasma. ${ }^{2}$ Plasmapheresis with fresh frozen plasma replacement has significantly improved the survival rates of these patients. ${ }^{3}$ However, a subset of patients with acquired TTP requires very long-term plasma therapy to prevent fatal outcome and to achieve a sustained remission. In these patients, complementary treatments with immunosuppressive agents [corticosteroids, vincristine, cyclophosphamide, azathioprine, cyclosporine A, high-dose intravenous immunoglobulin (Ig), staphylococcal protein immunoabsorption] and splenectomy has been advocated. Despite all the therapeutic options available, about one third of the patients with acquired TTP develop multiple relapses or persistent disease. ${ }^{4}$ Furthermore, patients with high titers of antibodies against ADAMTS 13 may not respond to plasma exchange alone. ${ }^{5}$ Treatment of such refractory or relapsing TTP with Rituximab has been effective and has resulted in lasting remissions. ${ }^{6}$

There are different conditions associated with TTP. These include viral infections such as CMV, and HIV. ${ }^{7}$ Interferon associated TTP has rarely been reported, especially in patients with chronic myeloid leukemia. However, only 9 cases of TTP have been reported in patients with chronic hepatitis $\mathrm{C}$ after interferon administration. ${ }^{8-14}$

Here we describe a woman with chronic hepatitis $\mathrm{C}$ infection, genotype $1 \mathrm{~b}$, with a positive hepatitis C RNA PCR one month prior to her admission. She was treated with pegylated interferon therapy for a positive hepatitis $\mathrm{C}$ RNA PCR. However, she developed acute TTP while on pegylated interferon therapy. Initially refractory to plasmapheresis and steroids, she responded well to weekly rituximab and plasmapheresis with disappearance of ADAMTS 13 inhibitors. Her hepatic transaminase levels remained normal during her treatment with Rituximab. TTP in chronic hepatitis $\mathrm{C}$ patients on interferon treatment has been treated with plasmapheresis and steroid in six of the 9 earlier reported cases with relative success. ${ }^{9-11,14}$ Two of the nine patients had a fatal outcome. Successful treated of an initial episode of acute refractory TTP with Rituximab and plasmapheresis in a patient on interferon therapy for chronic hepatitis $\mathrm{C}$ has not been reported.

\section{Case Report}

A 48-year-old African American woman was sent to the emergency room by her primary care physician for severe thrombocytopenia with a platelet count of $19,000 / \mathrm{mL}$. On admission, her platelet count was $6000 / \mathrm{mL}$. She had a past medical history of chronic hepatitis $\mathrm{C}$, genotype $1 \mathrm{~b}$, for the past 5 years. She was being treated with peginterferon alfa $2 \mathrm{a}$ for one month prior to her admission for chronic hepatitis C. Her HCV RNA titre by polymerase chain reaction (PCR) was positive at that time. However, at admission her HCV RNA by PCR was undetectable.

Laboratory tests revealed a low hemoglobin of $8.8 \mathrm{gm} / \mathrm{dL}$ (11.7-16.1), severe thrombocytopenia with a platelet count of $6000(130,000$ $450,000 / \mathrm{mL})$, hematocrit of 25.5 (37-51\%) with a normal white blood cell count. Her prothrombin time and partial thromboplastin time were
Correspondence: Jen C. Wang, Division of Hematology/Oncology, Brookdale University Hospital Medical Center, Brooklyn, NY 11212, USA.

Tel. +1.718.2405519 - Fax: +1.718.240-6516.

E-mail: jcwang5@aol.com

Key words: thrombotic thrombocytopenic purpura, interferon, hepatitis C, Rituximab.

Conflict of interests: the authors declare no potential conflict of interests.

Received for publication: 25 July 2012.

Revision received: 18 December 2012.

Accepted for publication: 7 January 2013.

This work is licensed under a Creative Commons Attribution NonCommercial 3.0 License (CC BYNC 3.0).

CC Copyright N. Poddar and J.C. Wang, 2013

Licensee PAGEPress, Italy

Hematology Reports 2013; 5:e2

doi:10.4081/hr.2013.e2

normal. Her renal functions were normal with BUN of $14 \mathrm{mg} / \mathrm{dL}$ and serum creatinine of 0.80 $\mathrm{mg} / \mathrm{dL}$ ) Aspartate aminotransferase was $44 \mathrm{U} / \mathrm{L}$ (14-36 U/L), alanine aminotransferase was $35 \mathrm{U} / \mathrm{L}$ (9-52 U/L) with a normal alkaline phosphatase of 77U/L (38-125U/L). She had indirect bilirubinemia, a negative direct coombs test, a normal fibrinogen level, an elevated reticulocyte count of $3.6 \%(0.5-2.5 \%)$ with a very high LDH of $4650 \mathrm{IU} / \mathrm{L}$ (313-618 IU/L) and a very low haptoglobin of $<7 \mathrm{mg} / \mathrm{dL}$. Her hepatitis profile revealed a positive hepatitis $\mathrm{C}$ antibody with undetectable HCV RNA by Polymerase chain reaction. Peripheral smear showed evidence of microangiopathic hemolysis with plenty of schistocytes, polychromasia, nucleated red blood cells and severe thrombocytopenia (Figure 1). Bone marrow examination did not reveal any abnormality. CT scan of chest, abdomen and pelvis did not reveal any underlying malignancy. Assay of vWF protease activity using the Technozym ADAMTS 13 ELISA chromogenic test revealed a severe deficiency (Figure 2) with a value of less than $47 \mathrm{ng} / \mathrm{mL}$ which corresponded to less than $9 \%$ of the normal activity (normal value more than 530 $\mathrm{ng} / \mathrm{mL}$ ) and presence of vWF inhibitor with a value of 1.8 (normal value less than 0.4 Bethesda Equivalent Units, BEU).

She was diagnosed with TTP likely related to interferon treatment for her chronic hepatitis C. Plasma exchange with equal volume of fresh frozen plasma was initiated daily along with prednisone at $1 \mathrm{mg} / \mathrm{kg}$. Platelet count increased and was $80,000 / \mathrm{mL}$ (Figure 2 ) by the $10^{\text {th }}$ day of treatment. Her platelet count dropped and was $41,000 / \mathrm{mL}$ by day 14 and $7000 / \mathrm{mL}$ by day 18 despite treatment. Twice 
daily plasmapheresis was started without any improvement in her platelet count. Subsequent treatment with weekly vincristine for two weeks also did not reveal any durable response. In view of her refractory disease, immunosuppressive therapy with weekly Rituximab was initiated at a dose of 375 $\mathrm{mg} / \mathrm{m}^{2}$, along with once daily plasmapheresis and prednisone. After the second week of Rituximab her platelet count, LDH and reticulocyte count started improving. Her ADAMTS 13 activity improved to $288 \mathrm{ng} / \mathrm{mL}(25-50 \%$ of the normal activity) with an undetectable level of vWF inhibitor. Schistocytes gradually disappeared from the peripheral smear. By the $4^{\text {th }}$ week of Rituximab, her ADAMTS 13 activity improved further to $360 \mathrm{ng} / \mathrm{ml}$ with rise in platelet count to $82,000 / \mathrm{mL}$. She had a stable hematocrit and a near normal serum LDH. Her hepatitis C RNA titers remained undetectable throughout her treatment without any elevation of her liver transaminases. In total she received 76 days (96 sessions) of plasmapheresis. The last outpatient visit almost 6 months after the completion of treatment revealed a platelet count of more than $350,000 / \mathrm{mL}$ with a normal $\mathrm{LDH}$ and a peripheral smear with no evidence of schistocytes. She has been in remission up to the date of this report and with no evidence of her hepatitis $\mathrm{C}$ reactivation.

\section{Discussion and Conclusions}

Most adults with acquired acute TTP have autoantibodies. ${ }^{2}$ Immunoglobulin $\mathrm{G}$ antibodies that inhibit ADAMTS13 activity have been detected in acquired TTP2 ${ }^{2}$. These antibodies have been reported in 48 to $80 \%$ of patients with recurrent TTP, suggesting that their presence may be associated with relapse. ${ }^{2}$ Plasma exchange with fresh frozen plasma has decreased the mortality rate in acute TTP significantly from more than $90 \%$ to less than $25 \% .^{3}$ Patients who do not respond well to plasma exchange usually require other treatment modalities of treatment, usually corticosteroids in combination with plasmapheresis or immunosuppressive therapy.

$\mathrm{HCV}$ infection has been associated with various autoimmune phenomena including TTP. This has been reported in a retrospective Italian study of 265 patients. ${ }^{7}$ Interferons are proteins and have strong immunogenic properties. Thyroid disease, connective tissue disorders, hematological complications and vascular abnormalities has commonly been reported in patients treated with interferon therapy. 8,9 TTP with interferon therapy even though rare, has been most frequently reported in patients with chronic myelogenous leukemia on interferon treatment. Only nine cases of TTP in patients with chronic hepatitis $\mathrm{C}$ have been reported in literature after administration of interferon. Iyado et al. ${ }^{10}$ reported the first suspected case of interferon induced TTP in a 57 year old man with chronic hepatitis $\mathrm{C}$ after more than 20 weeks of recombinant interferon therapy. The patient died before he could be treated with plasmapheresis. Kitano et al. ${ }^{11}$ reported serial ADAMTS 13 level and its inhibitor level for the first time in a patient with chronic hepatitis $\mathrm{C}$ patient who was treated successfully with plasmapheresis. Apart from 2 deaths, the other seven patients responded to plasmapheresis very early. ${ }^{12-14}$ Lambert et al. ${ }^{14}$ reported a case of interferon related TTP in a patient with chronic hepatitis C. Unlike our patient, she responded to plasmapheresis very early and was not treated with Rituximab. In all the 9 cases, none of them were treated with Rituximab.

In our patient, the ADAMTS 13 level was

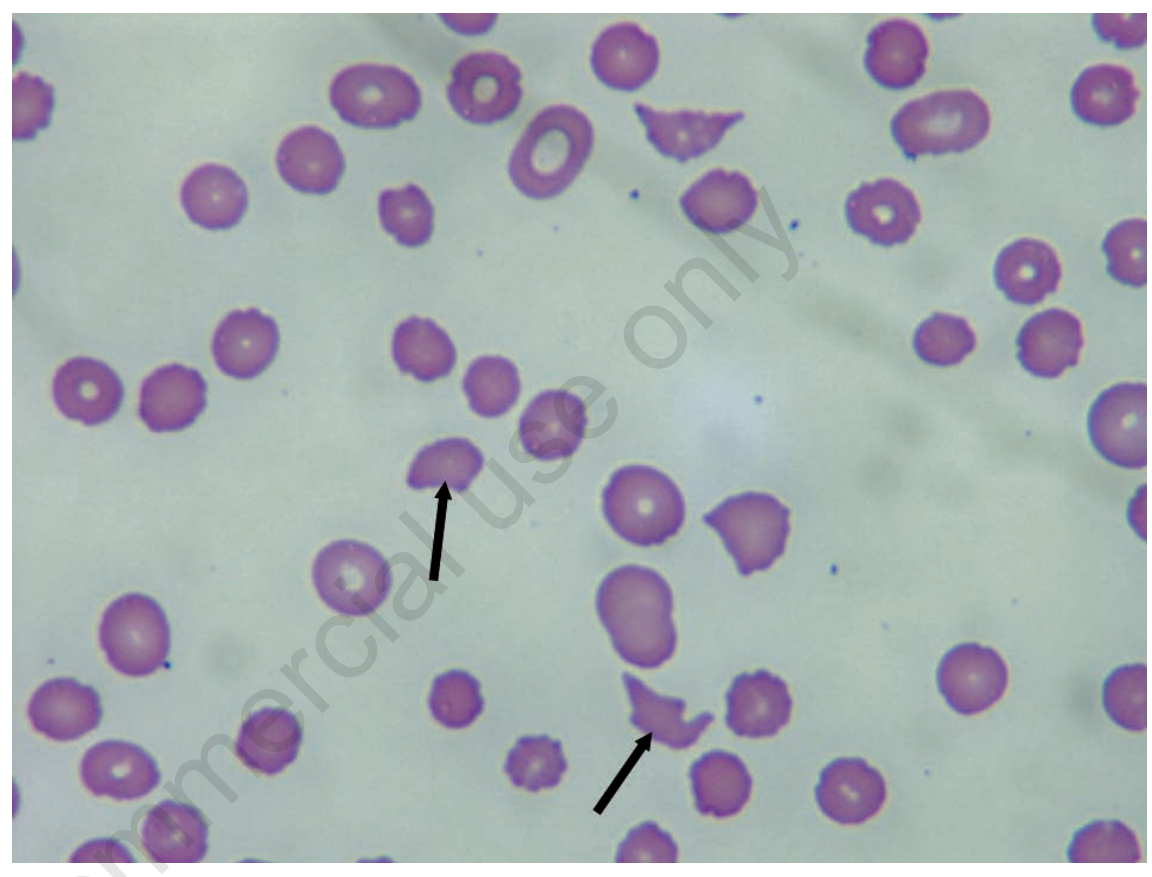

Figure 1. Peripheral smear showing plenty of schistocytes (indicated by arrow), polychromasia and markedly decreased platelets.

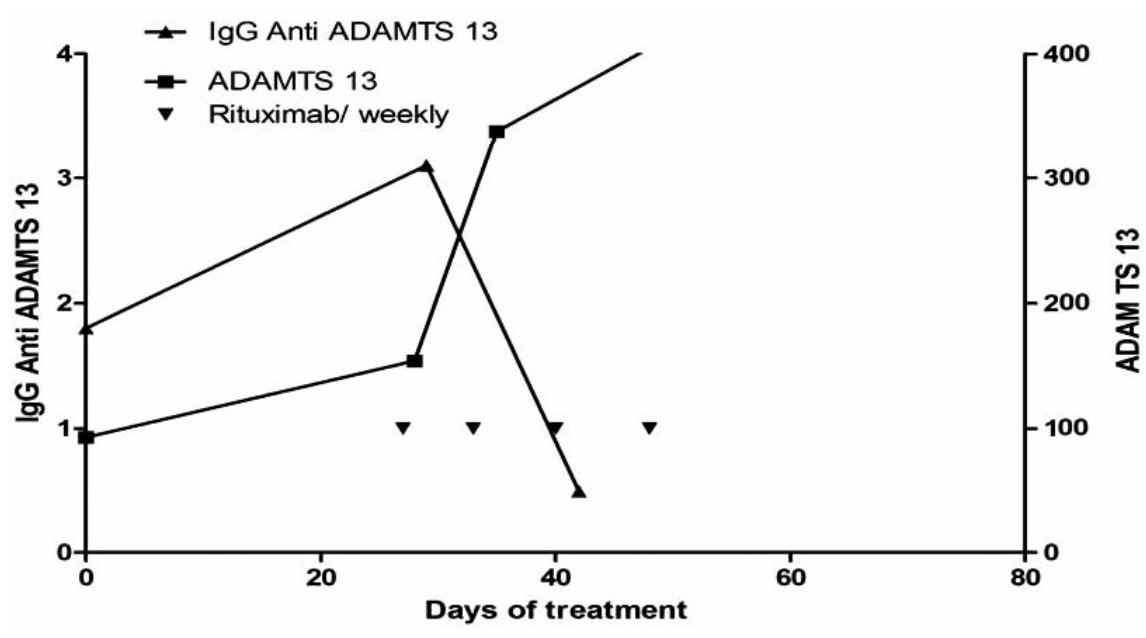

Figure 2. Time course of levels of ADAM TS $13(\mathrm{ng} / \mathrm{mL})$ and antibody to ADAM TS 13 (BEU) in relation to treatment with weekly Rituximab. 
markedly decreased with presence of inhibitor to ADAMTS 13 suggesting an acquired TTP. She did have a significant detectable viral load of HCV RNA one month prior to admission which responded very well and became undetectable on interferon therapy. Since there were no other medications notorious for the association with TTP and her hepatitis $\mathrm{C}$ was long standing for 5 years with negative mRNA PCR titers at admission, it is unlikely to be secondary to her hepatitis $\mathrm{C}^{12}$. Moreover, the onset of her TTP was after recent exposure to interferon treatment suggesting interferon as the likely cause of her TTP. It is possible that $\mathrm{HCV}$ and its treatment with interferon alter the immune system response and induce antiADAMTS13 antibody formation leading to an episode of TTP. Furthermore, initial treatment with plasmapheresis and steroid did not result in improvement of TTP. Plasmapheresis along with Rituximab ultimately resulted in a remission with undetectable VWF IgG inhibitor level and with improvement of ADAM TS 13 level. Hepatic transaminases and the Hepatitis C viral titers remained normal during the course of her treatment. An earlier case of TTP with membranoproliferative glomerulonephritis with Hepatitis C infection was successfully treated with plasmapheresis and Rituximab. However in that case patient was not treated with any interferon therapy in the past and treatment with Rituximab had resulted in the elevation of liver transaminase. ${ }^{15}$ Acute refractory TTP in a patient of chronic hepatitis $\mathrm{C}$, on interferon therapy, successfully treated with weekly Rituximab along with plasmapheresis and steroids during the first episode of TTP have not been reported previously in literature.

\section{References}

1. Moschcowitz E. Hyaline thrombosis of terminal arterioles and capillaries: A hitherto undescribed disease. Proc N Pathol Soc 1924;24:21-4.

2. Tsai HM. Advances in the pathogenesis, diagnosis and treatment of thrombotic thrombocytopenic purpura. J Am Soc Nephrol 2003;14:1072-81.

3. Rock GA, Shumak KH, Buskard NA, et al. Comparison ofplasma exchange with plasma infusion in the treatment of thrombotic thrombocytopenic purpura. Canadian Apheresis Study Group. N Engl J Med 1991;325:393-7.

4. Sadler JE, Moake JL, Miyata T, George JN. Recent advances in thrombotic thrombocytopenic purpura. Hematology 2004;407-23.

5. Tsai HM. High titers of inhibitors of von Willebrand factorcleaving metalloproteinase in a fatal case of acute thrombotic thrombocytopenic purpura. Am J Hematol 2002; 65:251-5.

6. Fakhouri F, Vernant JP, Veyradier A, et al. Efficiency of curative and prophylactic treatment with rituximab in ADAMTS 13-deficient thrombotic thrombocytopenic purpura: a study of 11 cases. Blood 2005;106:1932-7.

7. Giordano N, Amendola A, Papkostas P, et al. Immune and autoimmune disorders in HCV chronic liver disease: personal experience and commentary on literature. New Microbiol 2005;28:311-7.

8. Raanani P, Ben-Bassat I. Immune-mediated complications during interferon therapy in hematological patients. Acta Haematol 2002;107:13344.
9. Al- Zahrani H, Gupta V, Minden MD, et al. Vascular events associated with alpha interferon therapy. Leuk Lymphoma 2003; 44:471-5.

10. Iyoda K, Kato M, Nakagawa T, et al. Thrombotic thrombocytopenic purpura developed suddenly during interferon treatment for chronic hepatitis C. J Gastroenterol 1998;33:588-92.

11. Kitano K, Gibo Y, Kamijo A, et al. Thrombotic thrombocytopenic purpura associated with pegylated-interferon alpha-2a by an ADAMTS13 inhibitor in a patient with chronic hepatitis C. Haematologica 2006;91:ECR34.

12. Sallée $M$, Crétel $E$, Jean $R$, et al. Thrombotic thrombocytopenic purpura complicating interferon therapy in chronic C hepatitis. Gastroenterol Clin Biol 2008; 32:145-6.

13. Wilson LE, Widman D, Dikman SH, Gorevic. Autoimmune disease complicating antiviral therapy for hepatitis $\mathrm{C}$ virus infection. Semin Arthritis Rheum 2002;32: $163-73$.

14. Lambot F, Hanson B, Sztern B. Thrombotic thrombocytopenic purpura mediated by an ADAMTS13-inhibitor related to a treatment with pegylatedinterferon alpha-2a and ribavirine in a patient with chronic hepatitis C. Presse Med 2010;39:1207-10.

15. Mak SK, Lo KY, Lo MW, et al. Refractory thrombotic thrombocytopenic purpura and membranoproliferative glomerulonephritis successfully treated with rituximab: a case associated with hepatitis $\mathrm{C}$ virus infection. Hong Kong Med J 2009;15:201-8. 